\title{
AN IMBEDDING PROBLEM
}

\author{
J. W. CANNON AND S. G. WAYMENT
}

ABStract. If $H$ is an uncountable collection of pairwise disjoint continua in $E^{n}$, each homeomorphic to $M$, then there exists a sequence from $H$ converging homeomorphically to an element of $H$. In the present paper the authors show that if $\left\{M_{i}\right\}$ is a sequence of continua in $E^{n}$ which converges homeomorphically to $M_{0}$ and such that for each $i, M_{i}$ and $M_{0}$ are disjoint and equivalently imbedded, then there exists an uncountable collection $H$ of pairwise disjoint continua in $E^{n}$, each homeomorphic to $M$. For $n=2,3$, and $n \geqq 5$ it is shown that one cannot guarantee that the elements of $H$ have the same imbedding as $M_{0}$.

Introduction. Let $M$ be a continuum in $E^{n}$. It is well known that if $H$ is an uncountable collection of pairwise disjoint continua in $E^{n}$, each homeomorphic to $M$, then there exists $M_{0} \in H$ and a sequence $\left\{M_{i}\right\}$ from $H$ such that the sequence $\left\{M_{i}\right\}$ converges homeomorphically to $M_{0}$, that is, for $\epsilon>0$ there exists $N$ such that $i \geqq N$ implies the existence of a homeomorphism $h_{i}$ of $M_{0}$ onto $M_{i}$ which moves no point more than $\epsilon$.

The following question is immediately raised: suppose $M_{0}$ is a continuum in $E^{n}$ and $\left\{M_{i}\right\}$ is a sequence of pairwise disjoint continua in $E^{n}$ such that $\left\{M_{i}\right\}$ converges homeomorphically to $M_{0}$ and for each $i, M_{i} \cap M_{0}=\varnothing$. Does there necessarily exist an uncountable collection $H$ of disjoint continua in $E^{n}$ such that each $M^{\prime}$ in $H$ is homeomorphic to $M_{0}$ ? We remark that similar questions are discussed in [4].

The purpose of this note is to answer the above question in the affirmative under the additional condition that the $M_{i}, i=0,1$, $2, \cdots$, are equivalently imbedded in $E^{n}$ and to note that the answer to the question is negative for $n=2,3$, and $n \geqq 5$ under the condition that the elements of $H$ have the same imbedding as $M_{0}$. Two continua $M_{1}$ and $M_{2}$ are said to be equivalently imbedded in $E^{n}$ provided there exists a homeomorphism of $E^{n}$ onto $E^{n}$ which carries $M_{1}$ onto $M_{2}$. Finally, we note that if a nontopological imbedding property is imposed on the collection $H$ in $E^{2}$, then the answer is again negative.

Received by the editors November 14, 1969.

AMS Subject Classifications. Primary 5422, 5425, 5478.

Key Words and Phrases. Homeomorphic convergence, equivalently imbedded, $\epsilon$-homeomorphism, uncountable collection of continua. 


\section{The general problem in $E^{n}$.}

THEOREM 1. Suppose that the pairwise disjoint sequence of continua $\left\{M_{i}\right\}$ converges homeomorphically to $M_{0}$ in $E^{n}$ and suppose that for each $i, M_{i}$ and $M_{0}$ are disjoint and equivalently imbedded in $E^{n}$. Then there exists an uncountable collection $H$ of pairwise disjoint continua in $E^{n}$, each of which is homeomorphic to $M_{0}$.

Proof. The proof consists of constructing uncountably many sequences of homeomorphisms of $M_{0}$, each of which converges to a homeomorphism. The techniques of proof were first used by Bing [2], [4].

We remark that if $M$ and $M_{0}$ are equivalently imbedded, then there exists a homeomorphism $h$ of $E^{n}$ onto $E^{n}$ such that $h\left(M_{0}\right)=M$ and consequently the sequence $h\left(M_{i}\right)$ converges homeomorphically to $M$. We conclude that if $M$ and $M_{0}$ are equivalently imbedded, then for each $\epsilon>0$ there is a continuum $M^{\prime}$ disjoint from $M$ and a homeomorphism $h^{\prime}$ such that $h^{\prime}(M)=M^{\prime}, h^{\prime}$ moves no point of $M$ more than $\epsilon$, and $M^{\prime}$ and $M$ are equivalently imbedded. We shall refer to $h^{\prime}$ as a disjoint $\epsilon$-homeomorphism of $M$ onto $M^{\prime}$.

There exists a homeomorphism $f_{1}$ from $M_{0}$ onto $M_{1}$ and we shall choose $\epsilon_{1}$ so that $\rho\left(x, f_{1}(x)\right)<\epsilon_{1}$ for all $x \in M_{0}$. Let $f_{0}$ be the identity homeomorphism on $M_{0}$. For notational convenience let $f_{0}\left(M_{0}\right)=M^{0}$ and $f_{1}\left(M_{0}\right)=M^{1}$. If $E_{i}$ is the set of elements $\left(x_{1}, x_{2}\right)$ from $M_{0} \times M_{0}$ such that $\rho\left(x_{1}, x_{2}\right)>1 / i$, then it follows for any finite collection $K$ of homeomorphisms on $M_{0}$ that

$$
\inf _{\mathbf{x} \in K}\left\{\inf _{\left(x_{1}, x_{2}\right) \in E_{\boldsymbol{i}}}\left[\rho\left(k\left(x_{1}\right), k\left(x_{2}\right)\right]\right\}=\delta>0 .\right.
$$

Let

$$
\delta_{1}=\inf _{i=0,1}\left\{\inf _{\left(x_{1}, x_{2}\right) \in E_{1}}\left[\rho\left(f_{i}\left(x_{1}\right), f_{i}\left(x_{2}\right)\right]\right\},\right.
$$

let $\eta_{1}=\rho\left(M^{0}, M^{1}\right)$, and let $\epsilon_{1}^{\prime}=\min \left\{\delta_{1}, \eta_{1}, \epsilon_{1}\right\}$. For $\epsilon_{2}<\epsilon_{1}^{\prime} / 3$ there exist disjoint $\epsilon_{2}$-homeomorphisms $f_{1}^{0}$ and $f_{1}^{1}$ on $M^{0}$ and $M^{1}$ respectively. Let $f_{0}^{0}$ and $f_{0}^{1}$ be the identity homeomorphisms on $M^{0}$ and $M^{1}$ respectively, let $f_{j}^{i}\left(M_{i}\right)=M^{i j}$, for $i, j=0,1$, and let $f_{i j}\left(M_{0}\right)=f_{j}^{i}\left(M_{i}\right)$ $=f_{j}^{i}\left(f_{i}\left(M_{0}\right)\right)$ for $i, j=0,1$. Denote

$$
\inf _{i, j=0,1}\left\{\inf _{\left(x_{1}, x_{2}\right) \in E^{2}}\left[\rho\left(f_{i j}\left(x_{1}\right), f_{i j}\left(x_{2}\right)\right)\right]\right\}
$$

by $\delta_{2}$ and let $\eta_{2}=\min _{\left(i_{1}, i_{2}\right) \neq\left(j_{1}, j_{2}\right)}\left\{\rho\left(M^{i_{1}, i_{2}}, M^{j_{1}, j_{2}}\right)\right\}$ and let $\epsilon_{2}^{\prime}$ $=\min \left(\delta_{2}, \eta_{2}, \epsilon_{2}\right)$. To further simplify notation, let $\alpha(n)$ represent a finite sequence on the first $n$ positive integers into the set $\{0,1\}$, and 
for $\beta=0$ or 1 let $\alpha(n) \beta$ represent a finite sequence on the first $n+1$ integers into $\{0,1\}$ with $\beta$ being the value on $n+1$. Also, let $\alpha(\infty)$ represent a sequence on the positive integers into $\{0,1\}$. We next choose $\epsilon_{3}<\epsilon_{2}^{\prime} / 3$ and proceed to define $f_{1}^{\alpha(2)}$ to be disjoint $\epsilon$-homeomorphisms of $M^{\alpha(2)}$ and define $f_{0}^{\alpha(2)}$ to be the identity on $M^{\alpha(2)}$. Finally define $f_{\alpha(3)}\left(M_{0}\right)=f_{\beta}^{\alpha(2)}\left(M^{\alpha(2)}\right)$ for each $\alpha(2)$ and $\beta=0,1$. We continue the process inductively to obtain for each integer $n$, the $2^{n}$ homeomorphisms $f_{\alpha(n)}$.

Let $a$ be a number in $[0,1)$. If $a_{1} a_{2} \cdots=\alpha(\infty)$ represents the binary expansion of $a$ always chosen to not repeat ones infinitely and $\alpha(n)$ represents the $n$th truncated approximation, then the association of the sequence $\left\{f_{\alpha(n)}\right\}$ with $a$ is a one-to-one map from the interval $[0,1)$ into the collection of sequences previously constructed and defines an uncountable collection of sequences of homeomorphisms on $M_{0}$. The function of $f_{a}(x)=\lim _{n} f_{\alpha(n)}(x)$ is well defined since $\rho\left(f_{a}(x), x\right)$ $\leqq \sum_{i=1}^{\infty} \epsilon_{i}<\epsilon_{1} \sum_{i=0}^{\infty} 1 / 3^{i}=\epsilon_{1}(3 / 2)$. Since $f_{a}(\cdot)$ is defined on a compact subset of $E^{n}$, it follows from the usual advanced calculus argument on uniform convergence that $f_{a}(\cdot)$ is continuous. If $x_{1}$ and $x_{2}$ are distinct elements of $M_{0}$, then $\left(x_{1}, x_{2}\right) \in E_{n}$ for some $n$. Then $\rho\left(f_{\alpha(n)}\left(x_{1}\right), f_{\alpha(n)}\left(x_{2}\right)\right)$ $>\delta_{n}$. However,

$$
\rho\left(f_{\alpha(n)}\left(x_{i}\right), f_{a}\left(x_{i}\right)\right)<\sum_{i=n+1}^{\infty} \epsilon_{i}<\delta_{n} \sum_{i=1}^{\infty} 1 / 3^{i}=\delta_{n} / 2
$$

for $k=1,2$ and consequently, by the triangle inequality, $f_{a}\left(x_{1}\right) \neq f_{a}\left(x_{2}\right)$ and $f_{a}$ is one-to-one. We have shown that $f_{a}$ is a one-to-one continuous map from a compact metric space $M_{0}$ into a Hausdorff space $E^{n}$, and hence $f_{a}$ is a homeomorphism. We have left only to show that $f_{a}\left(M_{0}\right)$ and $f_{b}\left(M_{0}\right)$ are disjoint whenever $a \neq b$. Let.$a_{1} a_{2} \cdots$ and.$b_{1} b_{2} \cdots$ be the binary expansions of $a$ and $b$ and let $n$ be the first integer with $a_{n} \neq b_{n}$. Then $\rho\left(f_{\alpha(n)}\left(M_{0}\right), f_{\beta(n)}\left(M_{0}\right)\right) \geqq \eta_{n} \geqq \epsilon_{n}^{\prime}>3 \epsilon_{n+1}$. However, if $x \in M_{0}$ then

$$
\rho\left(f_{\alpha(n)}(x), f_{a}(x)\right)<\sum_{i=n+1}^{\infty} \epsilon_{i}<\epsilon_{n+1} \sum_{i=0}^{\infty} 1 / 3^{i}=\epsilon_{n+1}(3 / 2)
$$

and similarly $\rho\left(f_{\beta(n)}(y), f_{b}(y)\right)<\epsilon_{n+1}(3 / 2)$. Hence, again by the triangle inequality, $f_{u}\left(M_{0}\right) \cap f_{b}\left(M_{0}\right)=\varnothing$.

The problem in $E^{3}$. A homeomorphic image of the unit sphere is said to be vild or wildly imbedded in $E^{3}$ provided there is no selfhomeomorphism of $E^{3}$ which takes $S$ onto the unit sphere. The FoxArtin sphere $S_{0}$ (a wild sphere) has the property that there exists a 
sequence of disjoint tame spheres $\left\{S_{i}\right\}$ such that $\left\{S_{i}\right\}$ converges homeomorphically to $S_{0}$. It follows easily that there exists a sequence of disjoint Fox-Artin spheres $\left\{S_{i}\right\}$, each imbedded like $S_{0}$, such that $\left\{S_{i}\right\}$ converges homeomorphically to $S_{0}$ and such that $S_{i}$ $\operatorname{Cint}\left(S_{0}\right)$. Bing has shown [1], [3] that there do not exist uncountably many wild spheres in $E^{3}$. Thus if we consider the set $H$ of all spheres in $E^{3}$ imbedded equivalently with the Fox-Artin sphere, then there exists a disjoint sequence of elements from $H$ converging homeomorphically to an element from $H$ but no subset $H^{\prime}$ of $H$ can be composed of uncountably many pairwise disjoint elements. It is interesting to note that the existence of uncountably many disjoint spheres in $E^{3}$ follows from the existence of a sequence of Fox-Artin spheres converging to a Fox-Artin sphere by employing Theorem 1 . Of course the result also follows trivially by considering the set of all spheres of radius $r$ about the origin for $r$ in $(0,1]$.

The problem in $E^{2}$. A homeomorphic image $h(J)$ of the unit circle $J$ is said to be thick or thickly imbedded in $E^{2}$ provided the Lebesgue measure $\mu(h(J))>0$. A simple closed curve $J_{0}$ with positive Lebesgue measure is easily constructed and one can construct a sequence of disjoint thick homeomorphic images $J_{i}$ converging to $J_{0}$.

Let $H$ be the collection of all thick homeomorphic images of the unit circle $J$. Suppose $H$ contains an uncountable subset $H^{\prime}$ of pairwise disjoint elements. For each $i$, let $D_{i}$ represent the closed disk with radius $i$ centered at the origin. Then for some $n, D_{n}$ intersects each of uncountably many elements of $H^{\prime}$ in a set of positive measure. Hence for some $\epsilon>0, D_{n}$ intersects each of infinitely many elements of $H^{\prime}$ in a set of measure greater than $\epsilon$, which is impossible since the elements of $H^{\prime}$ are disjoint and the measure of $D_{n}$ is finite. Hence $H$ contains no uncountable subset of pairwise disjoint elements.

REMARK 1. An examination of the preceding technique shows that if $H$ is any collection of pairwise disjoint measurable sets in a $\sigma$ finite measure space, then at most a countable number of the elements from $H$ can have positive measure.

We note also that any homeomorphism of $E^{3}$ onto $E^{3}$ carries the set of wild spheres one-to-one and onto the set of wild spheres, while homeomorphisms of $E^{2}$ on to $E^{2}$ need not carry the set of thick Jordan curves onto the set of thick Jordan curves. This leads to a further sharpening of the question raised in the introduction.

Question. Let $M$ be a continuum in $E^{n}$ for $n \neq 1$ and let $H$ be the set of all homeomorphic images $h(M)$ of $M$ into $E^{n}$ such that $h(M)$ and $M$ are equivalently imbedded. Does the existence of a disjoint 
sequence of elements $\left\{M_{i}\right\}$ from $H$ such that $\left\{M_{i}\right\}$ converges homeomorphically to $M$ imply that there exists an uncountable pairwise disjoint subcollection $H^{\prime}$ of $H$ ?

It is known [5] that there does not exist an uncountable collection of $n$-cells in $E^{n}, n \geqq 5$, whose boundaries are pairwise disjoint and not flat. Using this fact and an argument similar to that given in the discussion of the problem in $E^{3}$ in this paper, one can answer the above question in the negative for $n=3$ and $n \geqq 5$. Examples 3 and 4 in [ 4 ] give a negative answer for $n=2$. The question remains unsettled for $n=4$.

\section{REFERENCES}

1. R. H. Bing, Conditions under which a surface in $E^{3}$ is tame, Fund. Math. 47 (1959), 105-139.

2. - - Each disk in $E^{3}$ contains a tame arc, Amer. J. Math. 84 (1962), 583-590. MR $26 \# 4331$.

3. - $E^{3}$ does not contain uncountably many mutually exclusive wild surfaces, Bull. Amer. Math. Soc. 63 (1957), 404. Abstract \#801t.

4. - Snake-like continua, Duke Math. J. 18 (1951), 653-663. MR 13, 265.

5. J. L. Bryant, Concerning uncountable families of $n$-cells in $E^{n}$, Michigan Math. J. 15 (1968), 477-479. MR 38 \#6561.

University of Wisconsin, Madison, Wisconsin 53706 and

Utah State University, Logan, Utah 84321 